\title{
1 Visible light-triggered NO generation from Naphthalimide-based probe for photoreceptor-mediated plant root growth regulation
}

\author{
3 Suprakash Biswas, ${ }^{1}$ Neha Upadhyay, ${ }^{2}$ Debojyoti Kar, ${ }^{2}$ Sourav Datta, ${ }^{* 2}$ Apurba Lal Koner*1 \\ $4 \quad{ }^{1}$ Department of Chemistry, Indian Institute of Science Education and Research Bhopal, Bhopal Bypass \\ 5 Road, Bhauri, Bhopal, Madhya Pradesh, INDIA \\ ${ }^{2}$ Department of Biological Sciences, Indian Institute of Science Education and Research Bhopal, \\ 7 Bhopal Bypass Road, Bhuari, Bhopal, Madhya Pradesh, INDIA
}

ABSTRACT: An efficient visible light-triggered nitric oxide (NO) releasing fluorescent molecule is designed and synthesized by coupling 2,6-dimethyl nitrobenzene moiety at the peri-position of 1, 8-naphthalimide through an alkene bond. The NO-releasing ability is investigated in details using various spectroscopic techniques, and the photoproduct was also characterized. Further, the photo-generated NO has been employed to examine the effect of photoreceptor-mediated NO uptake on plant root growth regulation.

Nitrogen monoxide is considered one of the most important signaling agents, exists in three interrelated chemical forms including nitrosonium cation $\left(\mathrm{NO}^{+}\right)$, nitric oxide $\left(\mathrm{NO}^{\circ}\right)$, and nitroxyl anion $\left(\mathrm{NO}^{-}\right){ }^{1-2}$ Nitric oxide (NO) exists as a highly reactive free radical or reactive oxygen species (ROS), sometimes also referred to as reactive nitrogen species (RNS), performs as an important multitasked signaling agent in various biological processes. ${ }^{3-5}$ NO has also been identified as an endothelium-derived relaxing factor (EDRF) in blood vessels and the nitric oxide synthase is mainly responsible for NO production in living systems. ${ }^{6-10}$ Besides, several diseases such as schizophrenia, Alzheimer's disease, and cancer are related to malfunction of NO signaling and its dynamics. ${ }^{11-13}$ For the past few decades, researchers also found that NO performs a crucial role in plant growth. ${ }^{14-15}$ In the metabolism of inorganic nitrogenous compounds in higher plants and nitrogen-fixing organism, NO is generated as a pivotal intermediate. ${ }^{16}$ Recent literature on plant biology reveals that NO influences primary root development through the initiation of cell-cycle genes and patterns of cellulose synthesis. ${ }^{17-18}$ Additionally, being an essential signaling agent, deficiency of NO affects auxin biosynthesis, transport, and signaling. ${ }^{19}$ The highly reactive nature of $\mathrm{NO}$ and its tremendous biological importance fascinated the researchers for in-situ generation of NO in biological systems. ${ }^{20-21}$ For potential therapeutic applications in living systems, the NO-donors must release NO in a time-controlled and site-specific manner. ${ }^{22-}$

${ }^{23}$ To date, numerous NO-donors such as 1-hydroxy-2-oxo-3-(aminoalkyl)-1-triazenes, 4-alkyl-2 hydroxyimino5-nitro-3-hexenes, etc. are reported in the literature. ${ }^{24-25}$ Among them, photo-triggered NO-donors secure extraordinary attention to the researchers due to the release of NO with high spatiotemporal control. Miyata group reported 6-Nitrobenzo[ $\alpha$ ] pyrene derivative that generates NO in the presence of visible light. ${ }^{26}$ Later, they reported a series of NO-releasing molecules, comprising of a hindered nitrobenzene derivative, but all 
36 from ultraviolet and two-photon excitation. ${ }^{28}{ }^{29-31}$ Therefore, there is an urgent need of a visible-light triggered

37 efficient NO-donor with considerable water-solubility.

In this contribution, we have designed and synthesized a naphthalene monoimide-based fluorescent molecule $\left(\mathrm{Ni}-\mathrm{NO}_{2}\right)$ for a visible light-induced $\mathrm{NO}$ generation. Thus, we have attached a sterically hindered nitrobenzene moiety, i.e., 2, 6-dimethyl nitrobenzene, at the peri-position of 1, 8-naphthalimide ring through an alkene spacer. Subsequently, elaborative spectroscopic investigations were performed to understand the visible lighttriggered NO release and further employed to comprehend the effect of NO in photoreceptor-mediated plant root growth regulation. The design strategy of $\mathrm{Ni}-\mathrm{NO}_{2}$ is based on photo-isomerization of nitro group of the

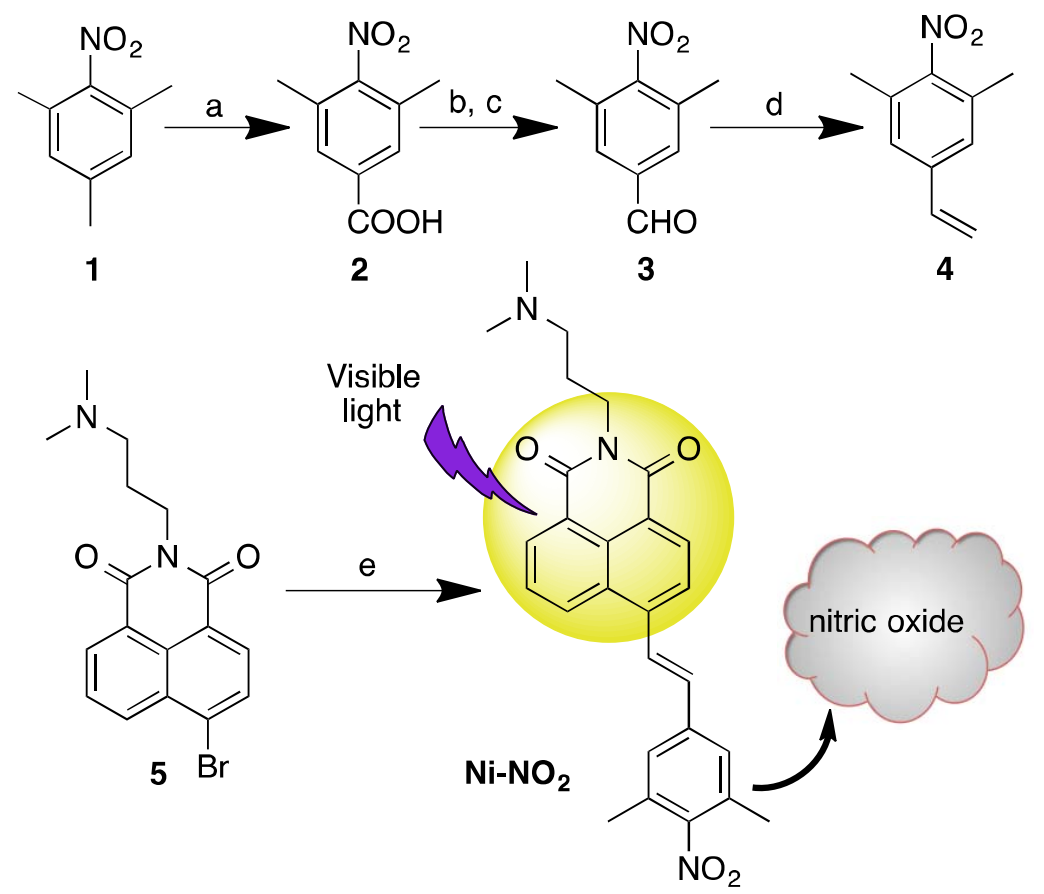

(a) $\mathrm{CrO}_{3}$, glacial $\mathrm{AcOH}, \mathrm{RT}, 10 \mathrm{~h}, 34 \%$; (b) $\mathrm{NaBH}_{4}, \mathrm{~F}_{3} \mathrm{~B}-\mathrm{OEt}_{2}$, dry THF, RT, $8 \mathrm{~h}, 67 \%$; (c) $\mathrm{MnO}_{2}$, dry DCM, Reflux, $6 \mathrm{~h}, 74 \%$; (d) $\mathrm{K}_{2} \mathrm{CO}_{3}$, dry THF, $\mathrm{Ph}_{3}{ }^{\odot}-\mathrm{CH}_{3} \mathrm{l}$, Reflux, $24 \mathrm{~h}, 80 \%$; (e) $4, \mathrm{Pd}(\mathrm{OAc})_{2}$, $\mathrm{P}(\mathrm{o}-\mathrm{tol})_{3}, \mathrm{ACN}-\mathrm{Et}_{3} \mathrm{~N}$, Reflux, $24 \mathrm{~h}, 44 \%$.

Scheme 1. Synthesis of $\mathrm{Ni}-\mathrm{NO}_{2}$ for visible-light triggered $\mathrm{NO}$ generation

sterically-hindered nitrobenzene moiety, connected to the naphthalimide ring. Considering the photoisomerization possibility, we have synthesized $\mathrm{Ni}^{-N_{2}}$ according to the above synthetic scheme (Scheme 1), the detailed synthetic scheme is shown in Scheme S1. Density functional study reveals that the nitro group is in non co-planar conformation with a twist angle of $c a .42^{\circ}$, with respect to the benzene ring due to the steric hindrance of two ortho-methyl groups (Scheme S2a-b and Fig. S1). Upon photo-irradiation, the twisted nitro group rearranges and isomerizes to nitrite ester, ${ }^{28}$ owing to the generation of NO (as shown in Scheme S2c).

After the synthesis and characterization, we validated the optical purity by comparing UV-Vis. and excitation spectra in phosphate buffer (PB, pH 6, Fig. 1a). $\mathrm{Ni}^{-N_{2}}$ has an absorption maximum in $\mathrm{PB}$ at around $400 \mathrm{~nm}$, whereas it emits around $517 \mathrm{~nm}$ (Fig. 1a) with a large Stokes shift of $117 \mathrm{~nm}$. To understand the swiftness 

under aCC-BY-NC-ND 4.0 International license.
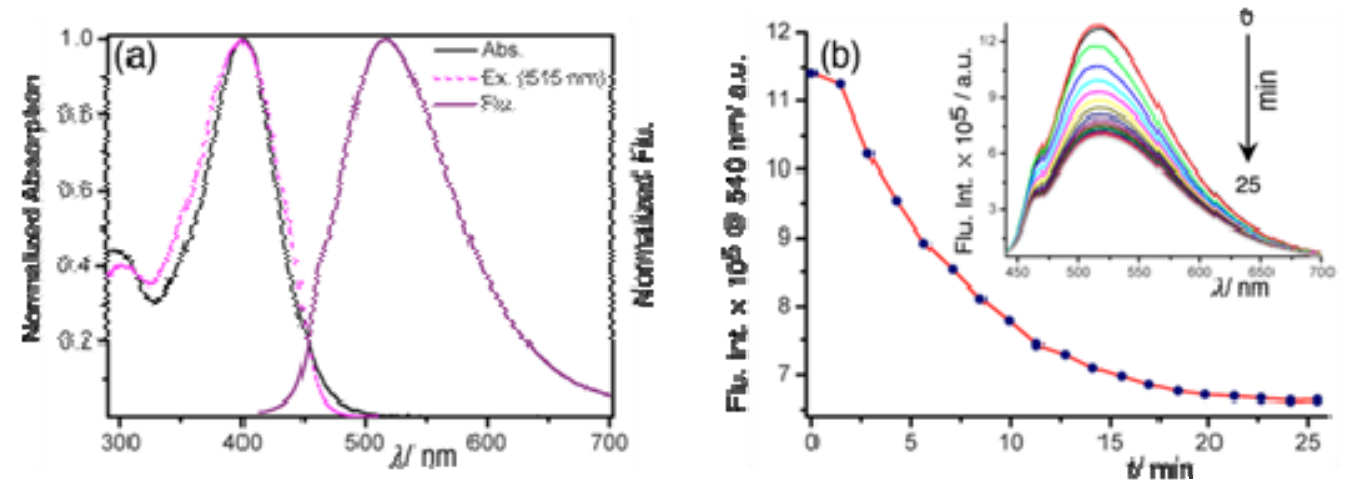

Fig. 1 (a) The absorption, excitation, and emission spectra of $\mathrm{Ni}^{-N_{2}}$ in $\mathrm{PB}$ at $\mathrm{pH} 6.0$, (b) kinetic monitoring of fluorescence intensity of $1 \mu \mathrm{M} \mathrm{Ni}-\mathrm{NO}_{2}$ at $540 \mathrm{~nm}$ in PB upon excitation of $410 \mathrm{~nm}$ light; inset shows fluorescence spectra of the same at different time interval

of NO-releasing properties of $\mathrm{Ni}-\mathrm{NO}_{2}$, we have kinetically monitored its fluorescence properties under visiblelight excitation (113 Lux) in $1 \mathrm{mM} \mathrm{PB}$ at $\mathrm{pH}$ 6. The fluorescence intensity of $\mathrm{Ni}-\mathrm{NO}_{2}$ gets attenuated upon visible-light mediated excited-state -isomerization of the nitro group. Interestingly, within 20 minutes of irradiation, we have obtained a plateau in the emission intensity indicating completion of NO release (Fig. 1b). The fluorescence quenching is possibly due to the formation of the phenolic group and its interaction with the solvent. ${ }^{32-33}$ Further, to verify the effect of light intensity on the NO release rate, we have performed kinetic monitoring of NO release experiment using a neutral density filter and recorded the emission spectra of $\mathrm{Ni}$ $\mathrm{NO}_{2}$. As expected, we obtained a plateau after $30 \mathrm{~min}$ (Fig. S2-3) confirming the role of light intensity for NO release. Furthermore, we have performed a modified Griess assay to validate NO generation through the photolysis of $\mathrm{Ni}_{-} \mathrm{NO}_{2} .{ }^{27}$ The photo-induced production of $\mathrm{NO}$ was verified by the detection of $\mathrm{NO}_{2}$, arising from the auto-oxidation of NO in PB medium. ${ }^{34}$ The appearance of red color solution having absorption at $542 \mathrm{~nm}$ supports the diazo- coupling reaction of $\mathrm{NO}_{2}{ }^{-}$with the Griess reagents (GR, Scheme S3). To perform this assay, we have taken $50-150 \mu \mathrm{M}$ of $\mathrm{Ni}_{-} \mathrm{NO}_{2}$ in $\mathrm{CHCl}_{3}$ and excited by $400 \mathrm{~nm}$ light for $\sim 50 \mathrm{~min}$, and then the mixture was allowed to react with GR. Finally, the absorption spectrum of the red color solution was measured. The increase optical density at $542 \mathrm{~nm}$ upon increasing concentration of $\mathrm{Ni}-\mathrm{NO}_{2}$, signifies the successful generation of NO (Fig. 2a). Further, to testify the photo-triggered generation of NO, an EPR experiment was also performed using 2-phenyl-4, 4, 5, 5,-tetramethylimidazoline-1-oxyl-3-oxide (PTIO) as a spin trap for NO. ${ }^{29,} 35$ After the photo-irradiation of a mixture of PTIO and $\mathrm{Ni}_{-} \mathrm{NO}_{2}$ for $30 \mathrm{~min}$ the EPR spectrum was recorded. 


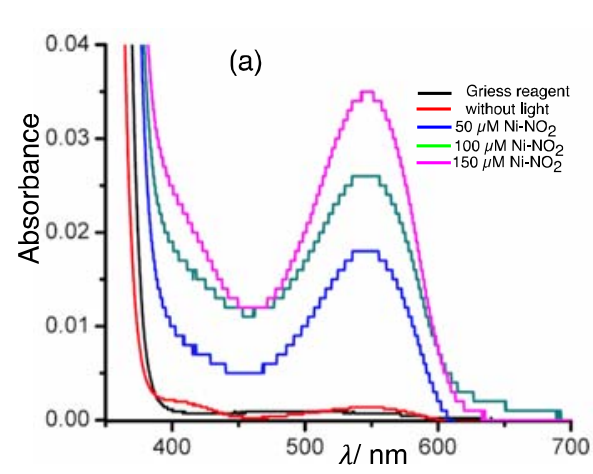

(b)

(c)

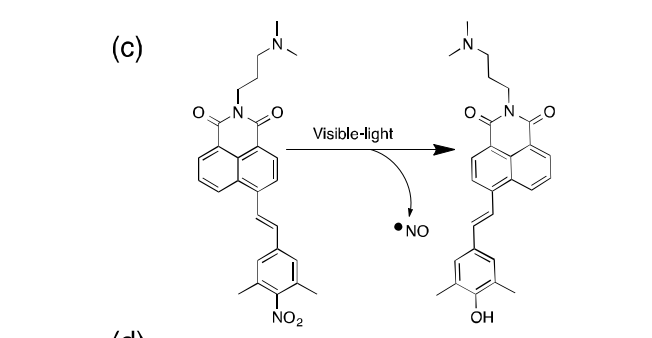

(d)

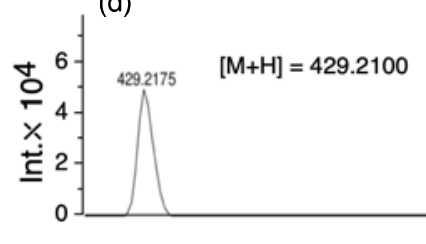

Fig. 2 (a) Absorption spectra of the reaction between Griess solution with a photo-irradiated $\mathrm{Ni}-\mathrm{NO}_{2}$ solution, (b) chemical structure of the photo-generated product of $\mathrm{Ni}^{-\mathrm{NO}_{2}}$ and (c) the mass-spectrum of the product after photodecomposition, (d) HPLC profile of photo-triggered NO generation experiments and (e) timedependent absorption spectra of $\mathrm{Ni}^{-\mathrm{NO}_{2}}(2 \mu \mathrm{M})$ in DMSO upon photo-irradiation using white light.

The characteristic EPR spectrum further confirms the generation of $\mathrm{NO}$ from Ni-NO $\mathrm{N}_{2}$ (Fig. S4). Additionally, we have performed a HPLC experiment to monitor the progress of the reaction. For HPLC measurement, we have irradiated $10 \mu \mathrm{M}$ of $\mathrm{Ni}_{-} \mathrm{NO}_{2}$ in acetonitrile using $390 \mathrm{~nm}$ light at $0,15,30,60$, and 80 min interval (see Fig. 2b and $\mathrm{SI}$ for details). A linear decrease in the absorption intensity corresponding to the reactant having an elution time 12.8 minute and subsequent increase in the absorption intensity for the product at an elution time of 4.6 minutes were observed (Fig. 2b). The photodecomposition quantum yield of NO release was measured as ca. 0.036 in acetonitrile (for details see SI, Fig. S5-7). The photo-degraded product was also identified by mass spectrometry (Fig. 2c-d). Further, the photo-reaction was also monitored by absorption spectroscopy in different time interval in DMSO (Fig. 2e) and in other solvents (see Fig. S8). The absorption spectra in DMSO reveal a decrease in the absorption maxima at $400 \mathrm{~nm}$ with the generation of a new absorption peak at around $427 \mathrm{~nm}$ which also confirms the formation of photo-generated product. ${ }^{33}$ 

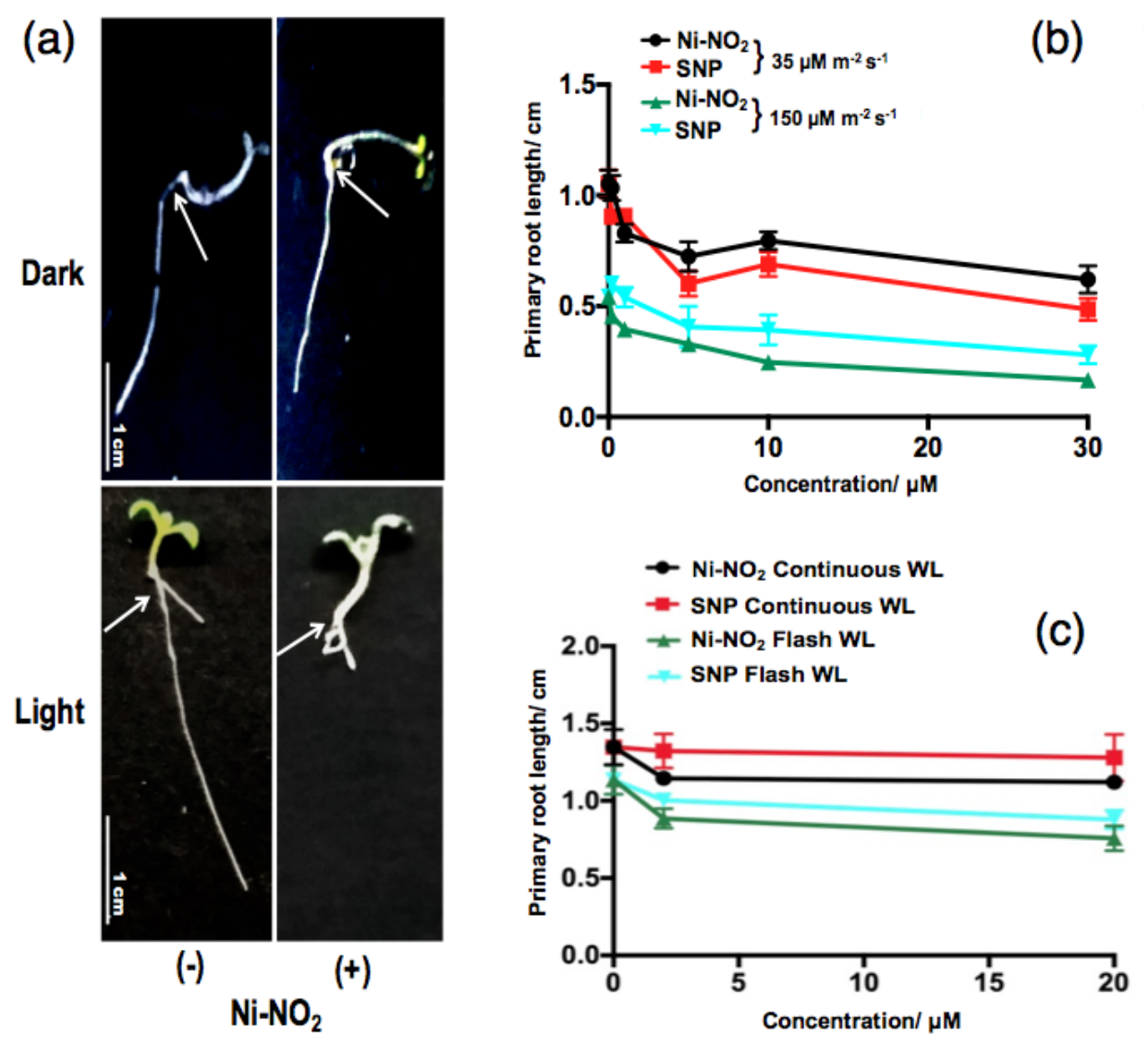

Fig. 3 Response of Arabidopsis (Col-0) roots to $\mathrm{Ni-NO}$, under dark and light. (a) 6 days old Arabidopsis seedlings grown on MS media plates supplemented with $0,10 \mu \mathrm{M} \mathrm{Ni-N_{2 }}$ in continuous dark and cycling white light at $110 \mu \mathrm{M} \mathrm{m}^{-2} \mathrm{~s}^{-1}$ fluence level. Part of seedling below white arrow shown in (a) indicates root that is inhibited by Ni-NO $\mathrm{N}_{2}$ in light due to the release of $\mathrm{NO}$. (b) Primary root length of Col-0 seedlings treated with 0 , (c) and in continuous white light $\left(110 \mu \mathrm{M} \mathrm{m}^{-2} \mathrm{~s}^{-1}\right)$ and flash of white light (110 $\mu \mathrm{M} \mathrm{m}^{-2} \mathrm{~s}^{-1}$ for 1 hour). Scale bar, $1 \mathrm{~cm}$ in (a).

After the spectroscopic characterization of the NO release in the presence of visible-light, we have investigated the effect of NO on plant roots and root-hair growth. A thorough literature survey suggests that NO inhibits root growth in a dose-dependent manner. ${ }^{36}$ Hence, to determine the effect of NO released from $\mathrm{Ni}-\mathrm{NO}_{2}$, we used Arabidopsis thaliana as a model plant and its root growth inhibition as a functional assay. Arabidopsis seeds (Col-0 ecotype) were inoculated on growth medium supplied with $0,0.2,1.0,5.0,10.0,30.0$ $\mu \mathrm{M}$ of $\mathrm{Ni}-\mathrm{NO}_{2}$. The NO-donor $\mathrm{Ni}-\mathrm{NO}_{2}$ was added inside the upper cover of plates at desired concentrations (Fig. S9). Seedlings were grown on $\mathrm{Ni}^{-\mathrm{NO}_{2}}$ free agar medium in the lower portion of the plate to avoid side effects of NO-donor reagents. ${ }^{37}$ One set of plates was kept under cycling white light $\left(110 \mu \mathrm{M} \mathrm{m}^{-2} \mathrm{sec}^{-1}\right)$ while the other set was kept under darkness. After six days seedlings grown under light and containing $\mathrm{Ni}-\mathrm{NO}_{2}$ in the upper lid showed significantly reduced primary root length as compared to the plate without $\mathrm{Ni}_{-} \mathrm{NO}_{2}$ (Fig. 3a). On the other hand, seedlings grown in the dark did not exhibit any significant difference in root length in the presence or absence of $\mathrm{Ni}-\mathrm{NO}_{2}$ (Fig. 3a). These results suggest that the photo-activation of $\mathrm{Ni}-\mathrm{NO}_{2}$ 

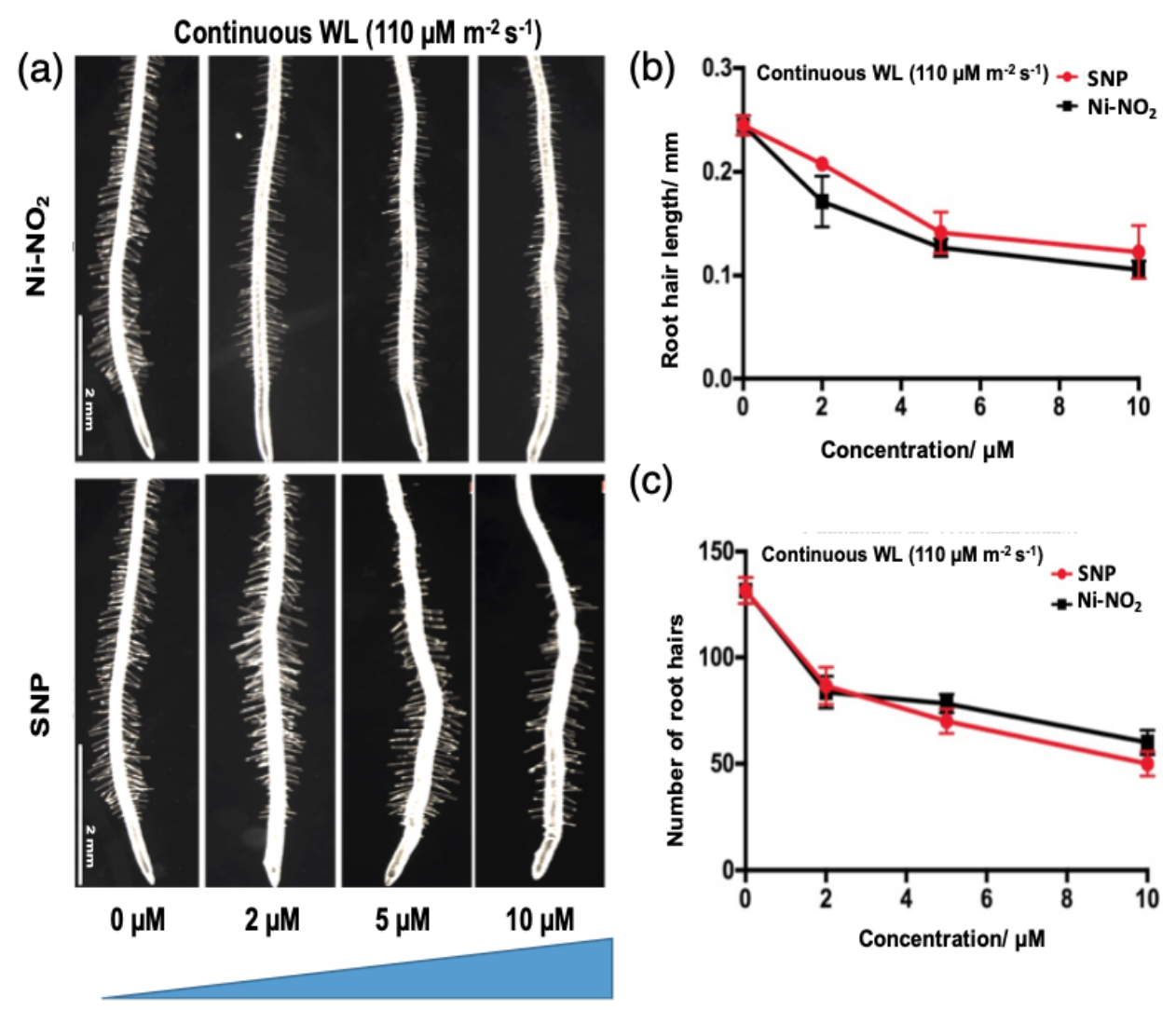

(c)

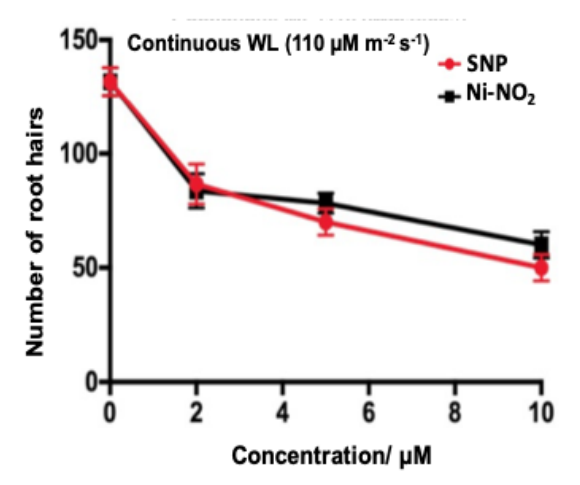

Fig. 4 Inhibition of root hair development by $\mathrm{Ni}_{-} \mathrm{NO}_{2}$ under white light. (a) Root hair image of 6 days old Col-0 seedlings grown on medium supplemented with $0,2,5,10 \mu \mathrm{M} \mathrm{Ni-NO}$ and SNP in continuous white light at $110 \mu \mathrm{M} \mathrm{m}^{-2} \mathrm{~s}^{-1}$ fluence level (b) Root hair length measurement of Col-0 seedlings treated as described in (a). (c) Root hair number measurement of Col-0 seedlings treated as described in (a). Mean values and S.E. were calculated from at least 30 seedlings. Scale bar, $2 \mathrm{~mm}$ in (a).

promotes the release of NO that causes root growth inhibition in plants. In order to evaluate the efficiency of $\mathrm{Ni}-\mathrm{NO}_{2}$ derived $\mathrm{NO}$ on root growth inhibition, we used sodium nitroprusside (SNP), a well-established NOdonor, and compared the effects on primary root growth inhibition (Fig. 3b-c). ${ }^{38}$ At similar concentrations of

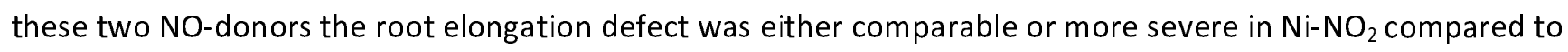
SNP (Fig. 3b-c). Further, we examined the effect of $\mathrm{Ni}^{-N_{2}}$ and SNP with varying fluence levels of cycling white light, 35, 85 and $150 \mu \mathrm{M} \mathrm{m}^{-2}$ sec $^{-1}$ (Fig. 3b and Fig. S10a-b). At all fluence levels, both the NO-donors showed reduced primary root length in a dose-dependent manner. We also determined the effect of continuous white light and flash of white light ( $1 \mathrm{~h}$ at $150 \mu \mathrm{M} \mathrm{m}^{-2} \mathrm{sec}^{-1}$ ) on NO-donors by using different doses of $\mathrm{Ni}-\mathrm{NO}_{2}$ and $\operatorname{SNP}(0,2$ and $20 \mu \mathrm{M})$ and exposing them to continuous or flash of white light. Both the light conditions exhibited an inhibitory effect on primary root growth (Fig. 3c and Fig. S11a, S12a). Further, we investigated the effect of monochromatic blue light on NO-donors by exposing plants to continuous blue light $\left(150 \mu \mathrm{M} \mathrm{m}^{-2} \mathrm{sec}^{-}\right.$ ${ }^{1}$ ) and a flash of blue light ( $1 \mathrm{~h}$ at $150 \mu \mathrm{M} \mathrm{m}^{-2} \mathrm{sec}^{-1}$, see $\left.\mathrm{SI}\right)$. Both Ni-NO${ }_{2}$ and SNP markedly inhibited primary root length under continuous blue light ( $\mathrm{SI}$, Fig. S11b, S12b). Under flash of blue light, $\mathrm{Ni}^{-\mathrm{NO}_{2}}$ at $2 \mu \mathrm{M}$ concentration promoted primary root length while at $20 \mu \mathrm{M}$ concentration it showed inhibitory effect on root growth (Fig. S12b, S13b). This might be due to cell elongation effect at a low concentration of NO under blue light. ${ }^{39}$ We also examined the effect of different concentrations $(0-10 \mu \mathrm{M})$ of $\mathrm{Ni}-\mathrm{NO}_{2}$ and SNP on root-hair 


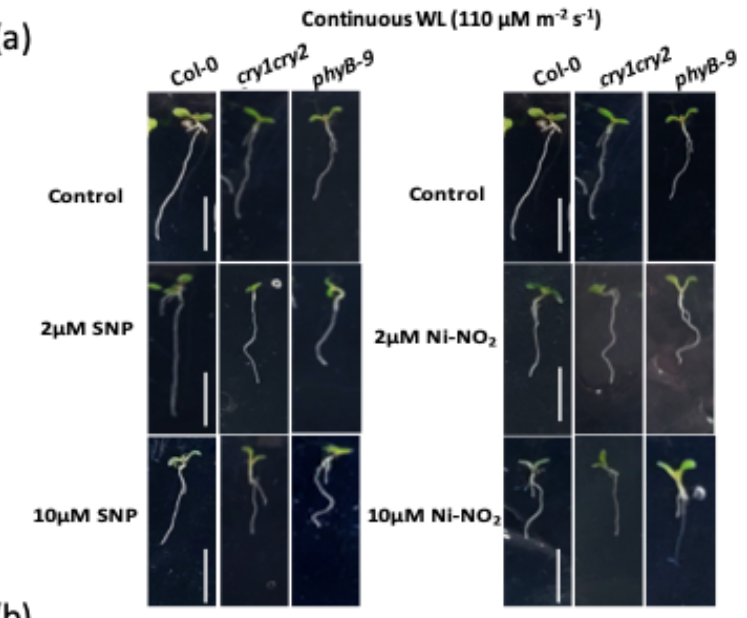

hair length and number) in a dose- dependent manner (Fig. 4a-c). This result suggests that the regulatory effect of $\mathrm{Ni}^{-\mathrm{NO}_{2}}$ on root growth of Arabidopsis seedlings is light dependent. Upon exposure to light, the photolysis of $\mathrm{Ni}-\mathrm{NO}_{2}$ releases $\mathrm{NO}$ which inhibits root growth. Light-mediated release of $\mathrm{NO}$ also activates various photoreceptors in plants. Phytochrome (PHY) and cryptochrome (CRY), red and blue light receptors respectively are reported to be NO-responsive. NO release causes nuclear accumulation of phyB-9 (b)

(a)

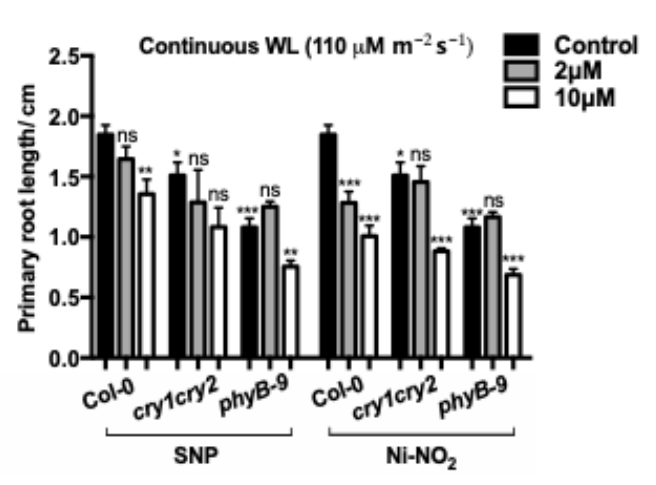

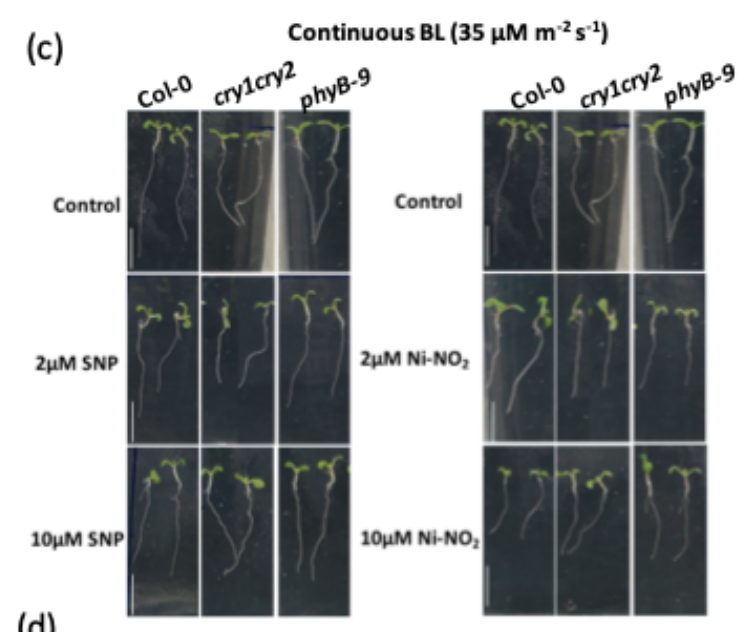

(d)

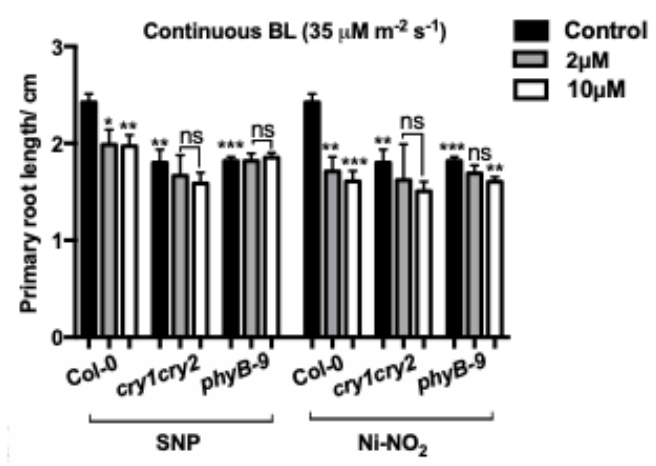

Fig. 5 Response of WT, cry1cry2 and phyB-9 mutants to NO under continuous white and blue light (a) WT, cry1cry2 and phyB-9 seedlings growing on medium supplemented with SNP and $\mathrm{Ni}^{-N_{2}}$ at various concentration under continuous WL at $110 \mu \mathrm{M} \mathrm{m}^{-2} \mathrm{~s}^{-1}$ fluence. (b) Root length of WT, cry1cry2 and phyB-9 seedlings treated as in (a). (c) WT, cry1cry2 and phyB-9 seedlings growing on medium supplemented with SNP and $\mathrm{Ni}-\mathrm{NO}_{2}$ at 0,2 and $10 \mu \mathrm{M}$ concentration under continuous blue light at $35 \mu \mathrm{M} \mathrm{m}^{-2} \mathrm{~s}^{-1}$ fluence. (d) Root length of WT, cry1cry 2 and phyB-9 seedlings treated as in (c). Mean value and SE were calculated from at least 20 seedlings. Significant difference was analyzed student's t-test with Welch's corrections compared to WT under same condition are indicated by asterisks: * $P<.05, * * P<.01, * * * P<.001$.

which positively regulates primary root growth inhibition. ${ }^{40}$ To verify, if the NO-induced primary root growth inhibition is photoreceptor mediated, we quantified root length in the photoreceptor mutants phy $B-9$ and cry1cry2 (Fig. 5 and S14-18). Our results indicate that phyB-9 and cry1cry2 exhibit hyposensitivity to the NO released by $2 \mu \mathrm{M} \mathrm{Ni}-\mathrm{NO}_{2}$. At $10 \mu \mathrm{M}$ concentration of $\mathrm{Ni}-\mathrm{NO}_{2}$ the root growth inhibition was also observed in the mutants especially under white light (WL) possibly due to the activation of other photoreceptors in the single mutant (Fig. 5a-b). Under the monochromatic blue light condition, cry1cry2 double mutantdisplay 
164 almost insensitivity at a higher concentration indicating that NO-induced primary root growth inhibition is 165 photoreceptor mediated (Fig. 5c-d).

166 In conclusion, we have designed and developed a naphthalene monoimide-based fluorescent NO-donor Ni$167 \mathrm{NO}_{2}$ by attaching 2,6 dimethyl nitrobenzene at the peri-position of 1,8-naphthalimide ring through an alkene 168 bond. The synthesized molecule releases NO upon photoexcitation with visible light and the NO generation 169 was confirmed by Griess assay, EPR spin trapping, HRMS and optical spectroscopic investigation in various solvents. Further, the progress of the reaction was monitored using HPLC. The photodecomposition quantum yield of $\mathrm{Ni}-\mathrm{NO}_{2}$ in acetonitrile was also determined. The fluorescence intensity of the probe gets quenched by

172 releasing NO in aqueous solution and its get saturated within 20 min signifying the rapid release of NO. Finally, 173 the released NO was employed for Arabidopsis root growth assay either by continuous or flash of white and 174 blue light irradiation. Our results indicate NO-mediated regulation of primary root and root hair growth by $\mathrm{Ni}-$ $175 \mathrm{NO}_{2}$ in a photoreceptor mediated manner. These findings can potentially open up prospects for generation of an effective weedicide in the future.

\section{Supporting Information}

Materials and methods, synthesis, photodecomposition QY measurement, kinetic study, DFT study, Griess assay, root growth experiments, characterization by UV-Vis., NMR, EPR and mass spectrometry.

\section{Corresponding Author}

*sdatta@iiserb.ac.in (SD) * akoner@iiserb.ac.in (ALK)

183

\section{ACKNOWLEDGMENT}

We acknowledge the financial support from IISER Bhopal (INGRANT/CHM/2012-017). SB thanks UGC, India for his fellowship. SD would like to thank DBT, India (Ramalingaswami Fellowship, IYBA) and SERB (EMR/2016/000181), India for funding. NU and DK acknowledge MHRD and DBT (BT/PR19193/BPA/118/195/2016), India for fellowship. Authors acknowledge Mr. Anil Raj Narooka for his help with HPLC measurements.

\section{REFERENCES}

1. Hughes, M. N., Relationships between nitric oxide, nitroxyl ion, nitrosonium cation and peroxynitrite. Biochim. Biophys. Acta 1999, 1411, 263-272.

2. Stamler, J. S.; Singel, D. J.; Loscalzo, J., Biochemistry of nitric oxide and its redox-activated forms. Science 1992, 258, 1898.

3. Domingos, P.; Prado, Ana M.; Wong, A.; Gehring, C.; Feijo, Jose A., Nitric Oxide: A Multitasked Signaling Gas in Plants. Molecular Plant 2015, 8, 506-520.

4. Stamler, J. S., Redox signaling: Nitrosylation and related target interactions of nitric oxide. Cell 1994, 78, 931-936. 
$199 \quad 5 . \quad$ Singh, S. K.; Husain, S. M., A Redox-Based Superoxide Generation System Using Quinone/Quinone 200 Reductase. ChemBioChem 2018, 19, 1657-1663.

2016 Förstermann, U.; Sessa, W. C., Nitric oxide synthases: regulation and function. E. Heart J. 2012, 33, $202829-837$.

203 7. Xia, Y.; Zweier, J. L., Direct measurement of nitric oxide generation from nitric oxide?synthase. Proc. 204 Natl. Acad. Sci. U.S.A. 1997, 94, 12705-12710.

205 8. Alderton, W. K.; Cooper, C. E.; Knowles, R. G., Nitric oxide synthases: structure, function and inhibition. 206 Biochem. J. 2001, 357, 593.

207 9. Palmer, R. M. J.; Ferrige, A. G.; Moncada, S., Nitric oxide release accounts for the biological activity of 208 endothelium-derived relaxing factor. Nature 1987, 327, 524.

209 10. Ignarro, L. J.; Buga, G. M.; Wood, K. S.; Byrns, R. E.; Chaudhuri, G., Endothelium-derived relaxing factor 210 produced and released from artery and vein is nitric oxide. Proc. Natl. Acad. Sci. U.S.A. 1987, 84, 9265.

211 11. Huang, F.-Y.; Chan Annie, O.-O.; Rashid, A.; Wong Danny, K.-H.; Cho, C.-H.; Yuen, M.-F., Helicobacter 212 pylori induces promoter methylation of E-cadherin via interleukin- $1 \beta$ activation of nitric oxide production in 213 gastric cancer cells. Cancer 2012, 118, 4969-4980.

214 12. Nikonenko, I.; Nikonenko, A.; Mendez, P.; Michurina, T. V.; Enikolopov, G.; Muller, D., Nitric oxide 215 mediates local activity-dependent excitatory synapse development. Proc. Natl. Acad. Sci. U.S.A. 2013, 110, 216 E4142.

217 13. Xiang, H.-J.; An, L.; Tang, W.-W.; Yang, S.-P.; Liu, J.-G., Photo-controlled targeted intracellular delivery 218 of both nitric oxide and singlet oxygen using a fluorescence-trackable ruthenium nitrosyl functional 219 nanoplatform. Chem. Commun. 2015, 51, 2555-2558.

220 14. Mangano, S.; Juárez, S. P. D.; Estevez, J. M., ROS Regulation of Polar Growth in Plant Cells. Plant 221 Physiol. 2016, 171, 1593-1605.

222 15. Domingos, P.; Prado, Ana M.; Wong, A.; Gehring, C.; Feijo, Jose A., Nitric Oxide: A Multitasked 223 Signaling Gas in Plants. Mol. Plant 2015, 8, 506-520.

224 16. Fewson, C. A.; Nicholas, D. J. D., Utilization of nitric oxide by micro-organisms and higher plants. $225 \quad$ Nature 1960, 188, 794-6.

226 17. Correa-Aragunde, N.; Graziano, M.; Chevalier, C.; Lamattina, L., Nitric oxide modulates the expression 227 of cell cycle regulatory genes during lateral root formation in tomato. J. Exp. Bot. 2006, 57, 581-588.

228 18. Correa-Aragunde, N.; Lombardo, C.; Lamattina, L., Nitric oxide: an active nitrogen molecule that 229 modulates cellulose synthesis in tomato roots. New Phytol. 2008, 179, 386-396.

230 19. Sun, H.; Feng, F.; Liu, J.; Zhao, Q., The Interaction between Auxin and Nitric Oxide Regulates Root 231 Growth in Response to Iron Deficiency in Rice. Front. Plant Sci. 2017, 8, 2169.

232 20. Wang, P. G.; Xian, M.; Tang, X.; Wu, X.; Wen, Z.; Cai, T.; Janczuk, A. J., Nitric Oxide Donors:? Chemical 233 Activities and Biological Applications. Chem. Rev. 2002, 102, 1091-1134. 
234 21. McCleverty, J. A., Chemistry of Nitric Oxide Relevant to Biology. Chem. Rev. 2004, 104, 403-418.

235 22. Carpenter, A. W.; Schoenfisch, M. H., Nitric oxide release: Part II. Therapeutic applications. In Chem.

236 Soc. Rev., The Royal Society of Chemistry: 2012; Vol. 41, pp 3742-3752.

237 23. Diring, S.; Wang, D. O.; Kim, C.; Kondo, M.; Chen, Y.; Kitagawa, S.; Kamei, K.-i.; Furukawa, S., Localized 238 cell stimulation by nitric oxide using a photoactive porous coordination polymer platform. Nat. Commun. 239 2013, 4, 2684.

24024 Davies, K. M.; Wink, D. A.; Saavedra, J. E.; Keefer, L. K., Chemistry of the Diazeniumdiolates. 2. Kinetics 241 and Mechanism of Dissociation to Nitric Oxide in Aqueous Solution. J. Am. Chem. Soc. 2001, 123, 5473-5481.

242 25. Napoli, C.; Ignarro, L. J., Nitric Oxide-Releasing Drugs. Annu. Rev. Pharmacol. Toxicol. 2003, 43, 97243123.

244 26. Fukuhara, K.; Kurihara, M.; Miyata, N., Photochemical Generation of Nitric Oxide from 6245 Nitrobenzo[a]pyrene. J. Am. Chem. Soc. 2001, 123, 8662-8666.

246 27. Suzuki, T.; Nagae, O.; Kato, Y.; Nakagawa, H.; Fukuhara, K.; Miyata, N., Photoinduced Nitric Oxide 247 Release from Nitrobenzene Derivatives. J. Am. Chem. Soc. 2005, 127, 11720-11726.

$248 \quad 28 . \quad K i t a m u r a$, K.; Kawaguchi, M.; leda, N.; Miyata, N.; Nakagawa, H., Visible Light-Controlled Nitric Oxide 249 Release from Hindered Nitrobenzene Derivatives for Specific Modulation of Mitochondrial Dynamics. ACS 250 Chem. Biol. 2016, 11, 1271-1278.

$251 \quad 29 . \quad$ Zhang, Z.; Wu, J.; Shang, Z.; Wang, C.; Cheng, J.; Qian, X.; Xiao, Y.; Xu, Z.; Yang, Y., Photocalibrated NO 252 Release from N-Nitrosated Napthalimides upon One-Photon or Two-Photon Irradiation. Anal. Chem. 2016, 88, $253 \quad 7274-7280$.

254 30. Xie, X.; Fan, J.; Liang, M.; Li, Y.; Jiao, X.; Wang, X.; Tang, B., A two-photon excitable and ratiometric 255 fluorogenic nitric oxide photoreleaser and its biological applications. Chem. Commun. 2017, 53, 11941-11944. photoinduced release of $\mathrm{HNO}$ or NO from an acyl nitroso compound, depending on environmental polarity. Chem. Commun. 2010, 46, 3788-3790. Jonusauskas, G., Controlling photophysics of styrylnaphthalimides through TICT, fluorescence and E,Zphotoisomerization interplay. Phys. Chem. Chem. Phys. 2017, 19, 1244-1256. 6, 7996-8000. 
269 36. Fernández-Marcos, M.; Sanz, L.; Lewis, D. R.; Muday, G. K.; Lorenzo, O., Nitric oxide causes root apical 270 meristem defects and growth inhibition while reducing PIN-FORMED 1 (PIN1)-dependent acropetal auxin 271 transport. Proc. Natl. Acad. Sci. U.S.A. 2011, 108, 18506.

272 37. Bai, S.; Yao, T.; Li, M.; Guo, X.; Zhang, Y.; Zhu, S.; He, Y., PIF3 Is Involved in the Primary Root Growth 273 Inhibition of Arabidopsis Induced by Nitric Oxide in the Light. Molecular Plant 2014, 7, 616-625.

274 38. Rico-Lemus, M.; Rodríguez-Garay, B., SNP as an Effective Donor of Nitric Oxide for in vitro Plant Cell 275 and Tissue Culture. J. Plant Biochem. Physiol. 2014, 2, e127.

276 39. Stöhr, C.; Stremlau, S., Formation and possible roles of nitric oxide in plant roots. J. Exp. Bot. 2006, 57, $277 \quad 463-470$.

278 40. Bai, S.; Yao, T.; Li, M.; Guo, X.; Zhang, Y.; Zhu, S.; He, Y., PIF3 is involved in the primary root growth 279 inhibition of Arabidopsis induced by nitric oxide in the light. Mol. plant 2014, 7, 616-625. 